\title{
A Survey on Trust Modeling from a Bayesian Perspective
}

\author{
$\operatorname{Bin} \operatorname{Liu}^{1,2}$ \\ 1 School of Computer Science, Nanjing University of Posts and Telecommunications \\ 2 Jiangsu Key Lab of Big Data Security \& Intelligent Processing \\ Email: bins@ieee.org
}

\begin{abstract}
In this paper, we are concerned with trust modeling for agents in networked computing systems. As trust is a subjective notion that is invisible, implicit and uncertain in nature, many attempts have been made to model trust with aid of Bayesian probability theory, while the field lacks a global comprehensive analysis for variants of Bayesian trust models. We present a study to fill in this gap by giving a comprehensive review of the literature. A generic Bayesian trust (GBT) modeling perspective is highlighted here. It is shown that all models under survey can cast into a GBT based computing paradigm as special cases. We discuss both capabilities and limitations of the GBT perspective and point out open questions to answer, with a hope to advance GBT to become a pragmatic infrastructure for analyzing intrinsic relationships among variants of trust models and developing novel tools for trust evaluation.
\end{abstract}

Keywords: Bayesian, networked computing systems, trust evaluation, trust modeling

\section{Introduction}

In the past decade, we have witnessed the advent of a variety of networked computing systems. Examples include wireless sensor networks (WSNs), internet of things (IoT), electronic commerce (EC), P2P networks, cloud computing, mobile ad hoc networks (MANETs), cognitive radio networks (CRNs), multiagent systems, semantic web, social networks, web-based recommender systems. 
A basic feature of such systems is the exploitation of the collective power of the network nodes to accomplish novel tasks.

In tandem with advances in network technologies, this has spurred a lot of renewed interest in trust modeling and evaluation. Generally speaking, trust is a concept with regard to the expectation, belief, and confidence on the integrity, reliability, security, dependability, ability, and other characters of an entity. It is a key factor that influences collaboration, competition, interaction and information sharing among network peers, and an enabling technology for decision making that facilitates the achievement of a system or application goal. We will use the words agents, entities, nodes, principals or peers interchangeably through the rest of this paper, as we will do with interactions, relationships, and links.

Trust has been studied for a long time [1]. The starting point of these studies may originate from the social sciences, for which trust between humans and its effects in economic transactions are the focus [2, 3, 4]. The notion of trust is intuitively easy to comprehend, while it has not yet been formally defined. Various definitions of trust have been proposed in different computing fields. Most of them depend on the context in which trust is investigated or the viewpoint adopted. Here are some examples as follows.

"Trust is the subjective probability by which an individual, A, expects that another individual, $\mathrm{B}$, performs a given action on which its welfare depends. " [5]

"Trust is the extent to which one party is willing to depend on something or somebody in a given situation with a feeling of relative security, even though negative consequences are possible." [1]

Trust is "a belief that is influenced by the individual's opinion about certain critical system features." [6]

"Trust (or, symmetrically, distrust) is a particular level of the subjective probability with which an agent assesses that another agent 
or group of agents will perform a particular action, both before he can monitor such action (or independently of his capacity ever to be able to monitor it) and in a context in which it affects his own action." [5]

"Trust is a psychological state comprising the intention to accept vulnerability based upon positive expectations of the intentions or behavior of another." [7]

"Trust is the willingness of the trustor (evaluator) to take risk based on a subjective belief that a trustee (evaluatee) will exhibit reliable behavior to maximize the trustor's interest under uncertainty (e.g., ambiguity due to conflicting evidence and/or ignorance caused by complete lack of evidence) of a given situation based on the cognitive assessment of past experience with the trustee." [8]

"Trust is a phenomenon that humans use every day to promote interaction and accept risk in situations where only partial information is available, allowing one person to assume that another will behave as expected." [9]

In addition to existing scattered definitions of trust, models for expressing trust involved in networked systems also lack coherence and consistency. Examples of the main formal techniques for modeling trust include fuzzy logic 10, 11, 12, 13, 14, 15, 16, 17, 18], subjective logic 19, 20, 21, 22, 23, 24, 25, 26, 27, 28, 29], Dempster-Shafer theory 30, 31, 32, 33, 34, 35], ratings [36, 37, 38, 39, 40], weighting 441, 42, 43, 44, 45], neural network [46, 47], Bayesian networks [48, 49, 50, 51], game theory 52, 53], swarm intelligence [54, 55, 56, 57], credential and policy 58, 59, 60, 61, 62, 63, 64, 65, 66], and others [67, 68, 69, 70, 71, 72, 42, 73, 74, 75].

Despite the discrepancy in definitions of and modeling tools for trust, it is well recognized that trust is important for promoting quick responses to a crisis, reducing transaction costs, avoiding harmful conflict, and enabling cooperative 
behavior. Trust can be regarded as a measure of a peer's faithfulness. It can also be treated as a prediction of the future behaviors of the peer who provide service. The trust value is thus a probability that a peer behaves satisfactorily in future interactions. When multiple aspects of the peer's interaction with other peers are considered, a trust vector, instead of a single value, can be used. Each element of this vector is used for evaluating one aspect. Most of the existing work on trust is highly specific to application considered. The theoretical underpinning of trust modeling and evaluation is important for creating a more cumulative body of knowledge on trust, while it remains a hard nut to crack.

In this paper, we focus on a Bayesian perspective for trust modeling for agents in networked computing systems. In spite of a few attempts to model trust using Bayesian techniques [76, 77, 78, 79, 80, 81, 82, 83, 84, 85], the field lacks a global overview for variants of Bayesian trust models and a discussion on their connections between each other. We present a study to fill in this gap. This paper does not pretend to be an exhaustive bibliography survey, but rather will review a gallery of selective research. A comprehensive bibliography can be found in other survey papers such as 86, , 87, 88, 89, 8, 90, 91, 92, 93, 94, 95, 96, 97, 98].

The major contributions of of this paper are summarized as follows.

1. We provide a high-level generic Bayesian perspective, termed generic Bayesian trust (GBT) here, for trust modeling and evaluation;

2. We give a literature review of classic variants of Bayesian trust models and some alternatives which can cast into the GBT paradigm;

3. We identify the strengths and weaknesses of the GBT paradigm;

4. We make an attempt to provide an improved understanding of Bayesian philosophy as well as its capabilities and limitations in modeling trust.

\subsection{Organization of this paper}

The remainder of this paper is organized as follows. Section 2 presents the GBT perspective and basic mathematical tools required to implement it. Section 3 provides a literature review of trust models that leverage the Beta 
distribution and Dirichlet distribution as the cornerstone. The relationship between these models and the GBT perspective is also discussed. Section 4 reviews state-space based trust models and discusses their connections to the GBT perspective. Section 5 presents a literature review on the subjective logic based trust models, and discusses their relationships to the GBT perspective. Section 6 provides an overall comparison of all models under survey. Section 7 discusses the capabilities and limitations of the GBT perspective in trust modeling and points out a number of open research questions. Finally, Section 8 concludes the paper.

\section{The GBT Perspective for Trust Modeling and Evaluation}

The GBT perspective is based on a collection of ideas. Assume that a trustor principal is interested in but uncertain about the trust (or distrust) of a trustee principal in a specific context. The trustor can quantify its uncertainty as a probability for the quantity it is interested in, and as a conditional probability for observations it might make, given the quantity it is interested in. When data arrives, Bayes theorem tells the trustor how to move from its prior probability to a new conditional probability for the quantity of interest, also known as the posterior probability in the jargon of Bayesian statistics. In this view, trust (or distrust) is quantitatively measured with a probability value ranging from 0 to 1.

The major insight leveraged in the GBT perspective is that, in spite of the complexity regarding the concept of trust, it has two natural and intrinsic attributes, namely uncertainty and subjectivity. The GBT perspective takes into account of such attributes as fully as possible to grasp the essence of trust, rendering it compatible with and applicable across different application domains.

To begin with, let us consider an open-ended network, which consists of network agents and links among the agents. At any point in time, there may be new agents joining the network and/or some others leaving. Each agent is associated with a unique identity (ID) and may interact with some others at 
some point in time. When a pair of agents interact with each other, we say that there is a link between them. Such interactions may take place between different pairs of network agents over time. During an interaction between a pair of agents, one agent plays the role of the trustor, and the other is called trustee. We can regard the trustee as the agent obliged to provide a service, and trustor as that receiving this service. The notion of trust is interpreted as the trustor's expectation of a certain future behavior of the trustee based on first-hand experience in interacting with it in the past and other possiblely relevant information provided by third-party agents.

We use the random variable $\Theta$ to denote the probabilistic trust of the trustee from the point of view of the trustor and use $Y$ to represent data the trustor observes. Realizations of $\Theta$ and $Y$ are respectively denoted by $\theta$ and $y$. We consider data items sequentially and represent the data item arriving at the $k$ th time step by $y_{k}$. As usual, the symbol $t$ is used as the continuously valued time variable. The value of $t$ at the $k$ th time step step is $t_{k}$.

Taking a Bayesian perspective, we translate the trust inference problem to recursively calculate the degree of belief in $\theta_{k}$ taking different values based on data observed up to time step $k$, namely $y_{1: k} \triangleq\left\{y_{1}, \ldots, y_{k}\right\}$. Specifically, we need to derive the probability density function (pdf) of $\theta_{k}$ conditional on observations $y_{1: k}$. This pdf is termed the posterior and represented as $p\left(\theta_{k} \mid y_{1: k}\right)$ or $p_{k \mid k}$ in short. Denote the initial pdf, also known as the prior, of the trust variable by $p\left(\theta_{0} \mid y_{0}\right) \equiv p\left(\theta_{0}\right)$, where $y_{0}$ denotes the set of no measurements. Then, the posterior can be obtained by recursively running two stages, namely, prediction and update.

Suppose that the posterior at time step $k-1$, namely $p\left(\theta_{k-1} \mid y_{1: k-1}\right)$, is available. The prediction stage runs based on the Chapman-Kolmogorov equation, yielding the prior pdf of the $\theta$ associated with time step $k$, as follows

$$
p\left(\theta_{k} \mid y_{1: k-1}\right)=\int p\left(\theta_{k} \mid \theta_{k-1}\right) p\left(\theta_{k-1} \mid y_{1: k-1}\right) d \theta_{k-1}
$$


In the above equation, $p\left(\theta_{k} \mid \theta_{k-1}\right)$ is determined by a transition function

$$
\theta_{k}=\mathrm{f}_{k}\left(\theta_{k-1}, \mathrm{v}_{k-1}\right)
$$

where the function $\mathrm{f}_{k}$ characterizes the time-evolution law of $\theta$ from time $t_{k-1}$ to $t_{k}, \mathrm{v}$ is an independent and identically distributed (i.i.d.) noise sequence used for modeling the uncertainty underlying the evolution law.

Upon the arrival of the new observation $y_{k}$, the update stage is invoked to update the prior pdf via Bayes' rule as follows

$$
p_{k \mid k}=\frac{p\left(y_{k} \mid \theta_{k}\right) p\left(\theta_{k} \mid y_{1: k-1}\right)}{p\left(y_{k} \mid y_{1: k-1}\right)}
$$

where the denominator is termed normalizing constant defined as

$$
p\left(y_{k} \mid y_{1: k-1}\right)=\int p\left(y_{k} \mid \theta_{k}\right) p\left(\theta_{k} \mid y_{1: k-1}\right) d \theta_{k},
$$

The likelihood function $p\left(y_{k} \mid \theta_{k}\right)$ here quantifies the trustor's uncertainty on the observation $y_{k}$, given the trust value $\theta_{k}$. It is worth to note that the task here is to compute the expected value of the unknown $\theta_{k}$, for which we need to derive the posterior $p_{k \mid k}$, but it is not necessary to compute the likelihood for any particular value of $\theta_{k}$. Based on Bayesian principle, $p_{k \mid k}$ can be computed from $p_{k-1 \mid k-1}$ recursively as follows

$$
p_{k \mid k}=\frac{p\left(y_{k} \mid \theta_{k}\right) \int p\left(\theta_{k} \mid \theta_{k-1}\right) p_{k-1 \mid k-1} d \theta_{k-1}}{p\left(y_{k} \mid y_{0: k-1}\right)} .
$$

Except for a few special cases, there is no analytic closed-form solution to (5). Here we introduce an approximate method, namely particle filter (PF) [99], to solve (5). The PF plays the role of the major computational engine for implementing the GBT paradigm. The PF algorithm has been widely used for tracking dynamic target distributions [100, 99, 101, 102, 103, 104, 105]. Suppose that we have a set of weighted samples $\left\{\theta_{0: k-1}^{i}, \omega_{k-1}^{i}\right\}_{i=1}^{N}$ at time step $k-1$, which can be used to construct a discrete approximation of $p_{k-1 \mid k-1}$. The samples are 
drawn from a proposal density, namely $\theta_{0: k-1}^{i} \sim q\left(\theta_{0: k-1} \mid y_{1: k-1}\right)$, and the sample weights satisfies $\omega_{k-1}^{i} \propto p\left(\theta_{0: k-1} \mid y_{1: k-1}\right) / q\left(\theta_{0: k-1} \mid y_{1: k-1}\right), \sum_{i=1}^{N} \omega_{k-1}^{i}=1$. The proposal density $q\left(\theta_{0: k-1} \mid y_{1: k-1}\right)$ is chosen to factorize such that

$$
q\left(\theta_{0: k} \mid y_{1: k}\right)=q\left(\theta_{k} \mid \theta_{0: k-1}, y_{k}\right) q\left(\theta_{0: k-1} \mid y_{1: k-1}\right)
$$

Each sample corresponds to a trajectory of the trust values up to time step $k-1$. At time $k$, each trajectory, say the $i$ th one, extends by sampling $\theta_{k}^{i}$ from a proposal distribution $q\left(\theta_{k} \mid \theta_{k-1}^{i}, y_{k}\right)$ and then being weighted by

$$
\omega_{k}^{i} \propto \omega_{k-1}^{i} p\left(\theta_{k}^{i} \mid \theta_{k-1}^{i}\right) p\left(y_{k} \mid \theta_{k}^{i}\right) / q\left(\theta_{k}^{i} \mid \theta_{k-1}^{i}, y_{k}\right)
$$

The posterior density $p_{k \mid k}$ can then be approximated by the updated sample set as follows

$$
p_{k \mid k} \approx \sum_{i=1}^{N} \omega_{k}^{i} \delta_{\theta_{k}^{i}}
$$

where $\delta_{\theta}$ denotes the Dirac-delta function located at $\theta$.

Starting from $\left\{\theta_{k-1}^{i}, \omega_{k-1}^{i}\right\}_{i=1}^{N}$, we summarize an iteration of the algorithm as follows:

- Sampling step. Sample $\hat{\theta}_{k}^{i} \sim q\left(\theta_{k} \mid \theta_{k-1}^{i}, y_{k}\right), \forall i$;

- Weighting step. Set $\omega_{k}^{i}$ using (77), $\forall i$; Normalize them to guarantee that $\sum_{i=1}^{N} \omega_{k}^{i}=1$

- Resampling step. Sample $\theta_{k}^{i} \sim \sum_{j=1}^{N} \omega_{k}^{j} \delta_{\hat{\theta}_{k}^{j}}$, set $\omega_{k}^{i}=1 / N, \forall i$.

As presented above, using the GBT perspective we translate trust evaluation to be a Bayesian sequential inference problem. See Algorithm 1 for a pseudo-code to implement trust inference based on the GBT perspective. We will show in Sections 3-6 that several most classic trust models cast into the GBT perspective if we appropriately define the prior and the likelihood function. 


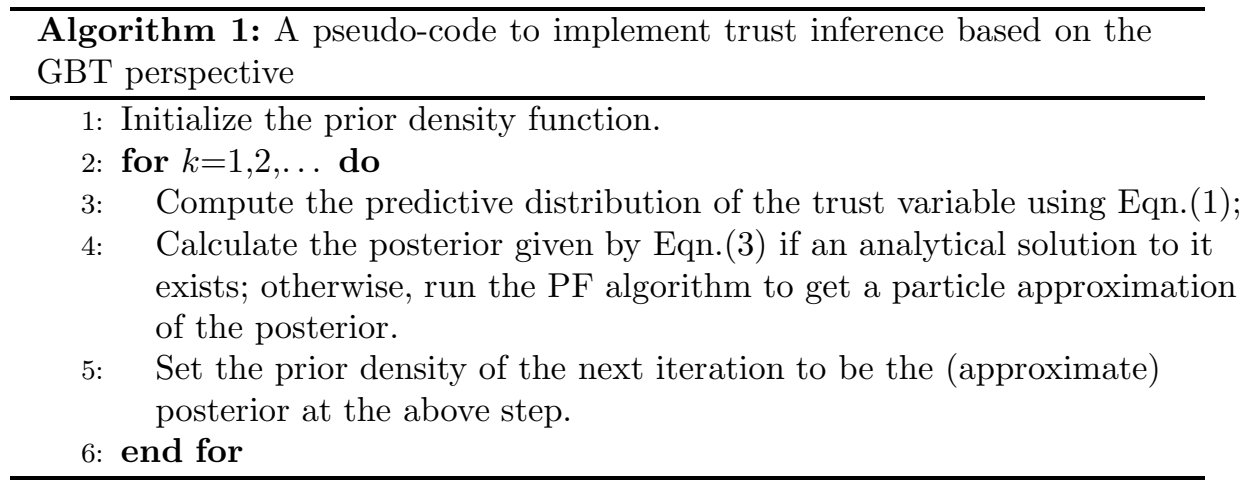

\section{Beta distribution and Dirichlet distribution based trust models}

This section presents a review of trust models that leverage Beta distribution and Dirichlet distribution models as major cornerstones. The relationships between these models and the GBT perspective presented in Section2 2 is discussed in Section 3.3 .

\subsection{Beta distribution based trust model (BDTM)}

As its name indicates, the basic idea of BDTM is using a parametric Beta model to represent the distribution of the probabilistic trust $\Theta$ [106, 107, 108, 109, 110]. The basic assumption leveraged by BDTM is that the outcome of each interaction is binary, e.g., \{success (good), failure (bad)\}. The trust variable $\Theta$ is interpreted as the probability that the outcome of an forthcoming interaction between the trustee and the trustor will be success (good). Therefore, a sequence of $n$ interactions $\mathbf{X}=X_{1}, \ldots, X_{n}$ is represented as a sequence of binomial (Bernoulli) trials and then modeled by a binomial distribution

$$
p(\mathbf{X} \text { consists of } m \text { successes })=\theta^{m}(1-\theta)^{n-m} .
$$

We can see that if the prior of $\Theta$ is represented by a Beta distribution $B(\alpha, \beta) \propto$ $\theta^{\alpha-1}(1-\theta)^{\beta-1}$, then the posterior $p(\Theta \mid \mathbf{X})$ will be $p(\Theta \mid \mathbf{X})$ is $B(\alpha+m, \beta+n-m)$, where $m$ denotes the number of successes in $\mathbf{X}, \alpha$ and $\beta$ are parameters of the 


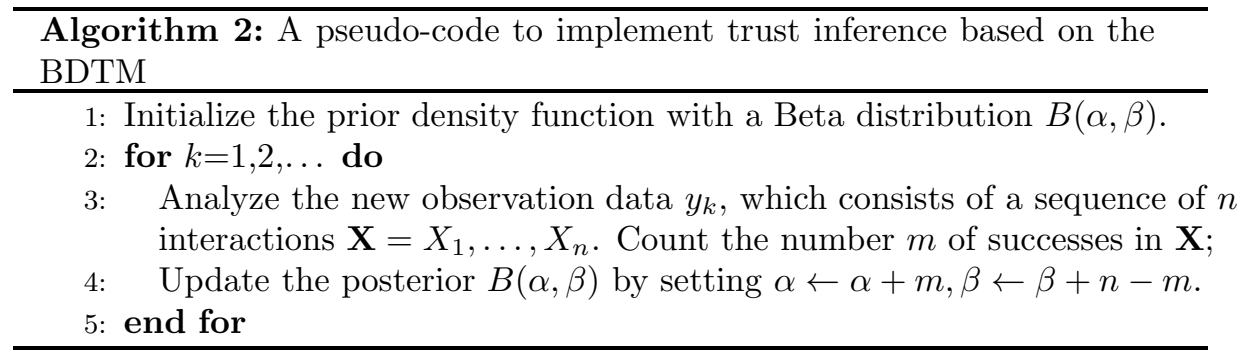

prior. See Algorithm 2 for a pseudo-code to implement trust inference based on the BDTM.

In real applications of BDTM, a disturbing issue may exist due to the socalled whitewashing problem, namely, the trustor has little or no previous experience in interacting with the trustee. A pragmatic solution to such problem is to allow the trustor to 'consult' about the trustee's behavior from third-party peers [11, 78], and then integrate its direct experience with information reported by third-party peers when assessing trust in the trustee.

A robust multiagent system is demanded to be able to deal with selfish, antisocial, or unreliable agents. A model presented in [111] characterizes the relationship between individual peer behaviors and group behaviors of peers through a two-layered hierarchy. Liu and Yang take into account of the possibility of that the third-party peers may be unreliable and thus reports provided by them may be inaccurate [78]. A notion, termed Advisor-to-Trustor Relevance (ATTR) metric, is proposed in [78] to quantitatively measure the systematic correlation of the subjectivity of the trustor and that of the third-party peer (i.e., the so-called advisor in [78]), based on the assumption that there is a common set of trustees, with which both the trustor and the advisor have interacted in the past. A report given by an advisor is weighted by its corresponding ATTR measure and then is used to update the posterior of $\Theta$ on the basis of BDTM [78].

To get rid of performance deterioration due to the presence of unfair rating provided by the advisors, specific data processing mechanisms are designed to filter unfair ratings that may result in misleading trust evaluations when us- 
ing BDTM [112]. One major mechanism is to consider recent ratings more by 'forgetting' old ratings as in the Bayesian reputation system (BRS) 19]. The basic assumption leveraged by BRS is that opinions provided by the majority of reputation sources are accurate, so any opinions deviating significantly from the average will be ignored. In contrast with BRS, the TRAVOS (Trust and Reputation model for Agent based Virtual OrganisationS) [107, 108] copes with unfair ratings by learning to distinguish reliable from unreliable third-party peers through repeated interactions with individual peers. However, TRAVOS relies on the assumption that the trustor and the third-party peers have extensive historical interactions that enable each third-party peer's expected honesty to be assessed. In addition, there is no time discounting for reports provided by third-party peers in TRAVOS. In contrast with TRAVOS, the Personalized Trust Model (PTM) 113] includes a forgetting factor to discount less recent ratings given by third-party peers.

\subsection{Dirichlet distribution based trust model (DDTM)}

The DDTM generalizes BDTMs with only 'binary' outcomes to multiple typed outcomes [114, 107, 115, 116, 117]. Such outcomes can be interpreted as different degrees of success on the 'success'-'failure' scale. In this case, $\Theta$ is in the form of a vector consisting of, say $b$ elements, viz. $\Theta \equiv\left[\Theta_{1}, \ldots, \Theta_{b}\right]$, with $\Theta_{i}$ representing the probability that the $i$ th type outcome in the $b$-way choice will happen in the next interaction. Correspondingly, the multinomial distribution is used to model a $n$-sequence of trials $\mathbf{X}$ with $b$ distinct outcomes. As a conjugate prior to multinomial trials, the Dirichlet distribution,

$$
D\left(\alpha_{1}, \ldots, \alpha_{b}\right) \propto \theta_{1}^{\alpha_{1}-1} \ldots \theta_{b}^{\alpha_{b}-1}
$$

is leveraged in DDTM, and then the posterior $p(\Theta \mid \mathbf{X})$ is also a Dirichlet distribution as follows

$$
D\left(\alpha_{1}+\sharp_{1}(\mathbf{X}), \ldots, \alpha_{b}+\sharp_{b}(\mathbf{X})\right),
$$




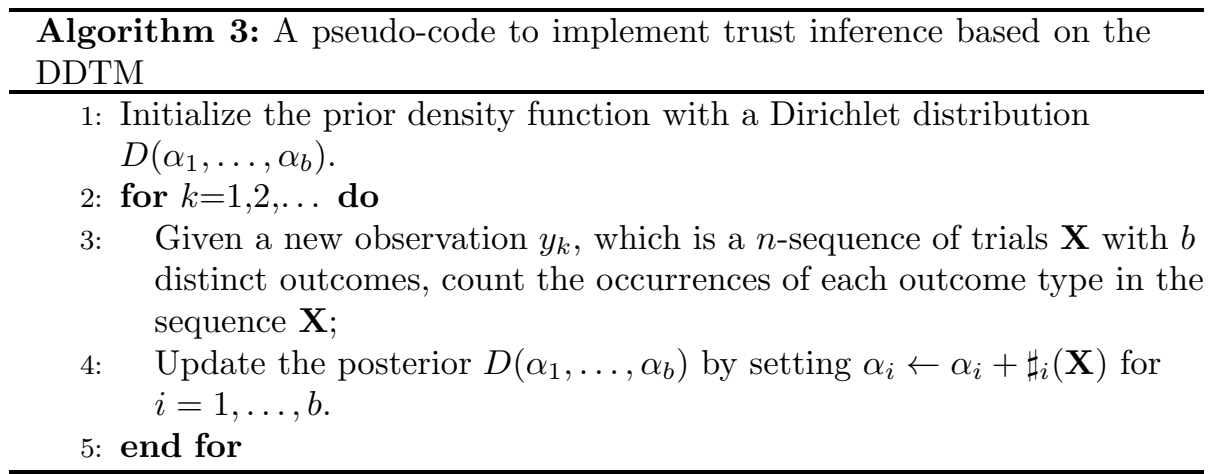

where $\sharp_{i}(\mathbf{X})$ counts the occurrences of the $i$ th type outcome in the sequence $\mathbf{X}$. See Algorithm 3 for a pseudo-code to implement trust inference based on the DDTM. The DDTM has been widely used in the context of global ubiquitous computing [116], especially E-Marketplaces [118].

\subsection{On relationships between $B D T M, D D T M$ and the $G B T$ perspective}

In this section, we clarify relationships between BDTM, DDTM and the GBT perspective.

If we define the state transition function (2) in GBT as follows

$$
\theta_{k}=\theta_{k-1}
$$

it leads to $p\left(\theta_{k} \mid \theta_{k-1}\right)=\delta_{\theta_{k-1}}$, and then $p\left(\theta_{k} \mid y_{1: k-1}\right)=p\left(\theta_{k-1} \mid y_{1: k-1}\right)$. That is to say that the prior pdf at time step $k$ is equivalent to the posterior at time step $k-1$. Now further restrict the initial pdf of $\theta, p\left(\theta_{0} \mid y_{0}\right)$, to be a Beta distribution, and then define the likelihood function $p\left(y_{k} \mid \theta_{k}\right)$ in (5) to be binomial as follows

$$
p\left(y_{k} \mid \theta_{k}\right)=\theta_{k}^{m}\left(1-\theta_{k}\right)^{n-m},
$$

where $n$ denotes the number of binary data items included in $y_{k}$ and $m$ the number of successes in $y_{k}$. Here $y_{k}$ is assumed to include multiple data items. Note that the GBT perspective allows any number of interactions to be performed between a pair of peers during a time interval. The resulting posterior (5) is 
then restricted to be a Beta distribution, due to the fact that the class of Beta prior distributions is conjugate to the class of binomial likelihood functions. Now the GBT framework reduces to the BDTM.

To summarize, the BDTM can be regarded as a special implementation case of the GBT framework, with specific definitions of the initial pdf $p\left(\theta_{0}\right)$ (i.e., in the form of Beta distribution), state transition function (as shown in (12)) and the likelihood (as shown in (13)).

Analogously, if we model the initial pdf $p\left(\theta_{0}\right)$ to be a Dirichlet distribution, specify the transition function in the same way as in (12) and represent the likelihood function to be multinomial, then the GBT framework reduces to the DDTM presented in Subsection 3.2. Therefore, the DDTM can also be regarded as an ad hoc implementation of the GBT framework.

In case of the trustee's past behavior data given by a third-party peer being available, the GBT framework allows the behavior data itself as well as the information on the third-party peer to be treated as a new observation that is then used to update the posterior pdf of $\Theta$. To handle such an observation using GBT, only an appropriate likelihood function is required to be set. Suppose that the Beta distribution model is under use and the trustee's past behavior data $\mathrm{X}$ reported by this third-party peer is an $n$-sequence of $m$ successes, then the likelihood function can be defined to be $\theta^{\theta_{t p p} m}, \theta^{\theta_{t p p}(n-m)}$, where $\theta_{t p p}$ denotes the trust value of this third-party peer from the point of view of the trustor. $\theta_{t p p}$ may be calculated based on past interactions between this third-party peer and the trustor. Then the resulting posterior of $\Theta$, after seeing this new observation, becomes $B\left(\alpha+\theta_{t p p} m, \beta+\theta_{t p p}(n-m)\right)$, assuming that the prior of $\Theta$ is $B(\alpha, \beta)$. If a series of new observations from multiple third-party peers are available, then the observations can be processed one by one, in the same way as illustrated above, and, correspondingly, the posterior pdf will be updated in a sequential manner.

Note that, for all cases discussed above, there is no need to apply the PF sampling approach to calculate the posterior pdf as shown in (5), because analytical solutions to (5) are available due to the adopted conjugate prior to the 
likelihood function.

\section{State-Space based trust model (SSTM)}

In contrast with the BDTM and DDTM that completely neglect the timeevolution feature of trust, the SSTM characterizes the time-evolution law of trust directly as follows [80, 77, 119]

$$
\theta_{k} \sim \mathcal{T}_{\mathcal{N}}\left(\alpha \theta_{k-1}, Q\right)
$$

where $\mathcal{T}_{\mathcal{N}}(m, Q)$ denotes a truncated normal pdf with mean $m$, variance $Q$ and support area $[0,1], 0 \leq \alpha \leq 1$ a forgetting factor specified by the model designer. An empirically setting of $\alpha$ is 0.85 given in [77]. In SSTM, the data $y$ is a continuously valued vector represented as $y_{k} \triangleq\left[y_{k, 0}, y_{k, 1}, \ldots, y_{k, n_{k}}\right]$, where $n_{k}$ denotes the number of neighbor (or similar) peers of the trustee at time step $k$. The first element $y_{k, 0}$ denotes the interaction outcome observed by the trustor when interacting with the trustee. The element $y_{k, i}, 0<i \leqslant n_{k}$, denotes the outcome observed by the trustor when interacting with the $i$ th neighbor peer of the trustee. Then the relationship between $\theta_{k}$ and $y_{k}$ is formulated via a likelihood function

$$
p\left(y_{k} \mid \theta_{k}\right)=\exp \left(\frac{-\left|\theta_{k}-V\right|}{\beta}\right)
$$

where $0<\beta<1$ controls the degree of the sensitivity of the likelihood value with respect to $V$. The term $V$ denotes the averaged voting value over all neighbor peers, namely,

$$
V \triangleq \frac{\sum_{i=1}^{n_{k}} U\left(i, y_{k}\right)}{n_{k}}
$$

in which

$$
U\left(i, y_{k}\right)= \begin{cases}1, & \text { if }\left|y_{k, i}-y_{k, 0}\right|<r \\ 0, & \text { otherwise }\end{cases}
$$

where $r$ denotes the maximum allowable difference between $y_{k, 0}$ and $y_{k, i}, i>0$ under the assumption that the trustee behaves in a trustworthy way. 


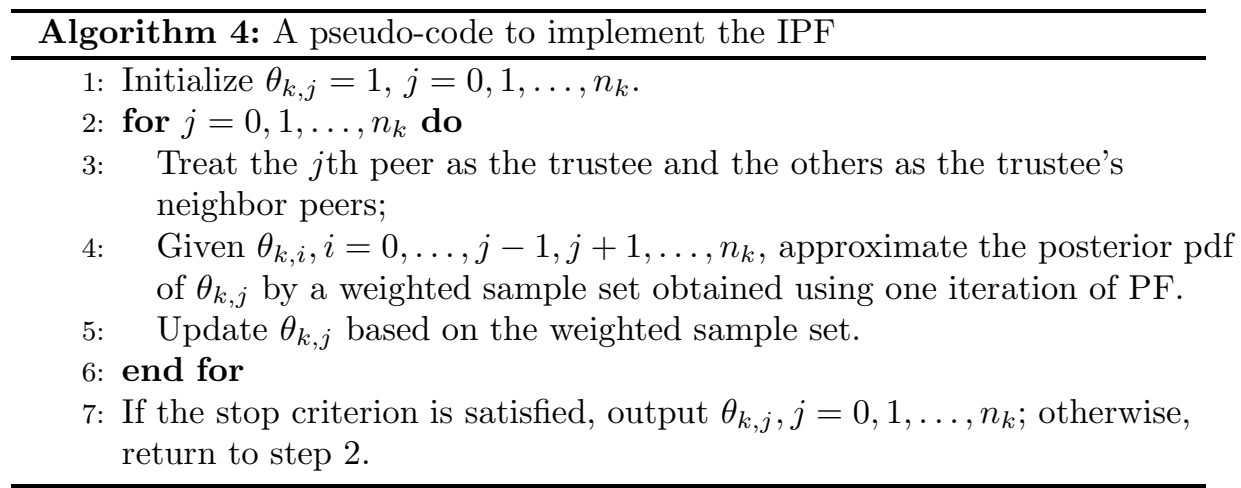

If $U\left(i, y_{k}\right)=1$, it can be interpreted as the $i$ th neighbor peer casting a vote of that the trustee is trusted. In the context of WSNs, the definition of $U\left(i, y_{k}\right)$ reflects a smooth variation in sensor readings of close-by reliable sensors [80, 77, 119]. A basic assumption leveraged here is that every neighbor peer of the trustee is totally trusted by the trustor. This assumption is relaxed in [80], in which an iterative PF method is presented that takes account of the trustworthiness of every neighbor peer in the calculation of $V$. Specifically, in that case, $V$ is defined to be

$$
V \triangleq \frac{\sum_{i=1}^{n_{k}} \theta_{k, i} U\left(i, y_{k}\right)}{\sum_{i=1}^{n_{k}} \theta_{k, i}}
$$

In a slight abuse of notation, here we use $\theta_{k, i}$ to denote the trust in the $i$ th neighbor peer of the trustee, and use $\theta_{k, 0}$ to denote the trust in the trustee; both are in the point of view of the trustor. It is worthy to note that every $\theta_{k, i}, i>0$ may be itself unknown. So an algorithm is required to estimate $\theta_{k} \triangleq\left[\theta_{k, 0}, \theta_{k, 1}, \cdots, \theta_{k, n_{k}}\right]$. An iterative PF (IPF) approach is proposed in [80] to estimate $\theta_{k, j}, j=0,1, \ldots, n_{k}$ one by one. The idea is to regard the $n_{k}+1$ network peers as members of a virtual committee. When estimating trust of a member in this committee, then this member is regarded as the trustee and the other members are regarded as neighbor peers of the trustee. A pseudo-code to implement IPF is presented in Algorithm 4 


\subsection{On relationship between $S S T M$ and the GBT perspective}

The relationship between SSTM and the GBT perspective is easy to check. If we set the state transition function (2) to be (14), and define the likelihood function $p\left(y_{k} \mid \theta_{k}\right)$ as (15), then the GBT based model reduces to the SSTM.

In comparison with BDTM and DDTM, SSTM is featured by its natural capability in characterizing the time-varying property of $\Theta$. This feature renders SSTM to be a better choice for characterizing dynamically changing sensing environments in many WSN applications such as robust sensor data fusion [77] and fault-tolerant event detection [119].

\section{Subjective logic based trust model (SLTM)}

Subjective logic operates on subjective beliefs about an event or subject of interest [120], wherein a belief is represented by the notion of opinion. In contrast with classical probability calculus that uses standard first-order probability representation, subjective logic employs second-order probabilities that is equivalent to a pdf over a first-order probability variable.

Assume that the trustor has an opinion on its trust in the trustee. Such an opinion is translated into degrees of belief or disbelief about the trustworthiness of the trustee in SLTM. The major feature of SLTM is that it quantitatively measures degrees of ignorance in a direct way. Borrowing the same notation from [120], we use $b, d$ and $i$ respectively to denote belief, disbelief, and ignorance. In SLTM, it is assumed that

$$
b+d+i=1,\{b, d, i\} \in[0,1]^{3},
$$

and an opinion on trust can be uniquely described by the triplet $\pi=\{b, d, i\}$. See Algorithm 5 for a pseudo-code to implement trust inference based on SLTM.

In 121], Ivanovska et al. present a method for computing the joint subjective opinion of multiple variables. In [122], Pope and Jøsang develop a subjective logic based approach to the evaluation of competing hypotheses. In [123], 


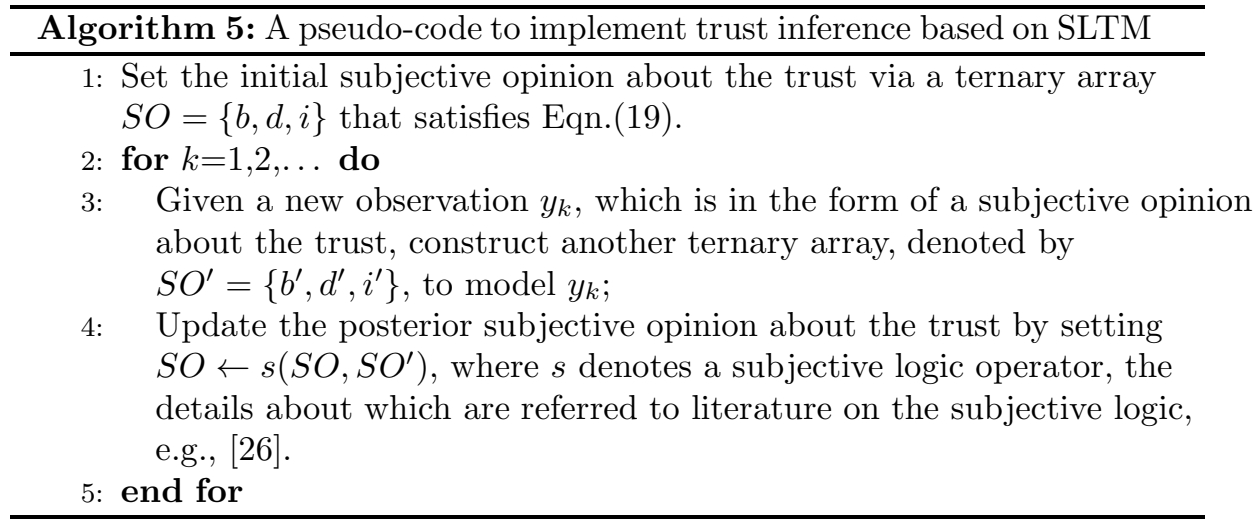

Ivanovska, Jøsang, and Sambo present an extension of Bayesian deduction to the framework of subjective logic. In [124], Jøsang focuses on the conditional reasoning in subjective logic, whereby beliefs are represented as binomial or multinomial subjective opinions. In [125] and [126], the authors address the issue of subjective logic based belief fusion, which consists of taking into account multiple sources of belief about a domain of interest. In particular, the article [125] discusses the selection of belief fusion operators and suggests to consider the nature of the situation to be modeled in searching the most appropriate one. The paper [126] describes cumulative and averaging multi-source belief fusion in the formalism of subjective logic, which represents generalizations of binarysource belief fusion operators. In [127], Jøsang and Kaplan introduce the term subjective networks, which generalizes Bayesian networks by letting it be based on subjective logic instead of probability calculus. In [29], Oren, Norman, and Preece introduce a subjective logic based argumentation framework, which is primarily used for evidential reasoning.

In [26], Jøsang applies SLTM to determine the trustworthiness of agents that are responsible for key generation and distribution in open networks. In 22], Alhadad et al. use subjective logic to express and deal with uncertainty in evaluating trust in a graph composed of paths that have common nodes. In [128, 129]. Jøsang et al. present an SLTM based method for trust network analysis. In [130], Liu et al. present a subjective logic based reputation model 
for blocking selfish behaviors in MANETs.

\subsection{On relationships between SLTM and GBT}

In this section, we discuss the connections between SLTM and GBT. As aforementioned, the most important feature of SLTM is that it allows to quantitatively measure the degree of ignorance in evaluating whether a trustee is trusted or not. It models belief $b$, disbelief $d$, and ignorance $i$ on the trustworthiness of the trustee by a triplet $\pi=\{b, d, i\}$, which satisfies (19). In concept, we can interpret $i$ as an intermediate state on the 'belief'-'disbelief' scale. Now let us suppose that, in the context of DDTM, the interaction outcomes between the trustor (or advisor) and the trustee can be represented by three degrees of belief on the trustworthiness of the trustee, namely 'belief' $b$, 'ignorance' $i$, and 'disbelief' $d$. Then the trust parameter can be represented as $\Theta \equiv[b, i, d]$. Let the prior of $\Theta$ follow a Dirichlet distribution $D\left(\alpha_{1}, \alpha_{2}, \alpha_{3}\right)$. Then, given an $n$-sequence $X$ of trials $\left(n=n_{1}+n_{2}+n_{3}\right)$ with 3 distinct outcomes, the posterior follows the Dirichlet distribution

$$
D\left(\alpha_{1}+n_{1}, \alpha_{2}+n_{2}, \alpha_{3}+n_{3}\right)
$$

where $n_{j}$ counts the occurrences of the $j$ th type outcome in the sequence $\mathbf{X}$. As is shown, a bijective mapping between subjective opinions leveraged in SLTM and evidence parameters of Dirichlet pdfs exists. This bijective mapping is confirmed in [125]. In 123], Ivanovska, Jøsang and Sambo also state that

"Every subjective opinion can be "projected" to a single probability distribution, called projected probability distribution which is an important characteristic of the opinion since it unifies all of its defining parameters."

This mapping has been exploited for translating from inference with subjective opinions to inference with a corresponding Dirichlet pdf [131, 132].

To summarize, DDTM can also be applied to measure the degree of ignorance in evaluating the trustworthiness of a trustee, in a similar spirit as SLTM. 
Table 1: A comparison of the model types under survey in terms of the data type to be processed, forms of the prior and the likelihood function adopted

\begin{tabular}{cccc}
\hline Model Class & type of observation & Prior & Likelihood \\
\hline BDTM & Binary & Beta & Binomial \\
DDTM & multiple typed & Dirichlet & multinomial \\
SSTM & Continuous & truncated normal & any form \\
SLTM & Three-valued & a ternary array $\{b, d, i\}$ & N $/$ A \\
GBT & any type & any form & any form \\
\hline
\end{tabular}

Table 2: Properties of Bayesian trust models

\begin{tabular}{ccccc}
\hline item & BDTM & DDTM & SSTM & GBT \\
\hline Conjugate prior required? & yes & yes & no & no \\
Support analytic inference? & yes & yes & no & depends \\
Explicitly model the evolution of trust? & no & no & yes & yes \\
application-specific? & yes & yes & yes & no \\
\hline
\end{tabular}

Since DDTM can be regarded as a specific ad hoc implementation of the GBT framework, as discussed in Section 3.3. the connections between SLTM and the GBT framework is built by using the DDTM as a bridge between them.

\section{An overall comparison of all model types under survey}

In this section, we attempt to give a big picture of relationships among the aforementioned model types. Table 1 lists the data types to be processed, forms of the prior and the likelihood function adopted, by each model type under consideration. In this table, we show that adopting the GBT perspective allows us to process any type of observations, either discrete or continuously valued, and adopt any form of prior or likelihood functions, in a completely Bayesian manner. This prominent feature of the GBT perspective can extend the application scope of Bayesian-based trust models to wider areas. A further comparison for model types within the Bayesian paradigm is presented in Table 2. It shows that, compared with BDTM, DDTM, and SSTM, GBT perspective is more flexible and generic. 


\section{Discussions on the GBT perspective}

In this section, we discuss the capabilities, limitations of the GBT perspective in trust modeling, and then point out several open research issues to answer in the way to advance it to become a pragmatic infrastructure for analyzing intrinsic relationships between variants of trust models and for developing novel ones for trust evaluation.

\subsection{On capabilities of the GBT perspective}

As presented in Sections 3.3 , 4.1, and 5.1 the GBT perspective provides a basic theoretical tie that connects BDTM, DDTM, SSTM, and SLTM together. It is shown that all these models cast into a common framework as special implementation cases of the GBT perspective. This is due to the natural power the Bayesian philosophy owns in uncertainty quantification and the inherent capabilities possessed by the GBT perspective, which will be discussed as follows.

\subsubsection{Capability to characterize the non-symmetry property of the trust}

It is an agreement in the literature that trust is not symmetric [9, 111], which implies that two peers involved in an interaction may not necessarily have the same trust in each other, even if they are presented with the same evidence. This is because the trustor and trustee may not necessarily interpret the outcome of each interaction in the same way [111].

The GBT perspective provides a natural way to take into account of such aforementioned non-symmetry in the interpretation of interaction outcomes. Consider again an interaction involving two agents 1 and 2. Denote the data yielded from this interaction as $y$. Then we can use two different likelihood functions, represented here as $p_{1 \rightarrow 2}\left(y \mid \theta_{2}\right)$ and $p_{2 \rightarrow 1}\left(y \mid \theta_{1}\right)$, to formulate the two different interpretations of $y$, from perspectives of agent 1 and 2 , respectively. Here $\theta_{1}$ and $\theta_{2}$ denote agent 2's trust in agent 1 and agent 1 's trust in agent 2, respectively. 


\subsubsection{Capability to take account of the dynamic property of the trust}

Some trust models, like TRAVOS, assume that the behavior of agents does not change over time [107], while in many cases agents may change their behavior over time actively or passively. In particular, some agents may have timebased behavioral strategies. The GBT perspective provides at least two simple mechanisms for taking account of the dynamic property of the trust. The first one is to characterize the time-evolution law of trust directly through the state transition function (2). It has been proved successful in a number of applications $[80,119,77]$. The other mechanism is to design a likelihood function that can reflect the effect of time on the trust value. For instance, in the likelihood function, we can assign each observation a weight, whose value depends on when this observation was generated. A similar strategy of downweighting out-of-date ratings is presented in [75].

\subsubsection{Capability to model the transitivity of trust}

The property of transitivity of trust is often employed in establishing trust relationships between a pair of peers that have never interacted before. In such situations, an intermediary peer, also known as an advisor or third-party peer, can help if we allow for transitive trust. The question needed to be addressed is that if the trustor trusts the advisor to some extent, and the advisor has past experience in interacting with the trustee, then to what extent the trustor should trust the trustee. This notion of transitive trust is very natural in many domains, e.g., when buyers of a product recommend this product to new buyers and audience of a movie recommend this movie to new audience. We can handle this transitivity issue flexibly and efficiently when adopting the GBT perspective. Suppose that an advisor provide historical data about the trustee to the trustor. From the trustor's point of view, the observation it receives consists of two parts, namely the trustor's trust $\theta_{a}$ in the advisor, and the historical data $X$ about the trustee. We can design an appropriate likelihood function $p\left(X \mid \theta, \theta_{a}\right)$ to take account of the transitivity issue, while the form of the likelihood function is dependant on application domains. Suppose that the BDTM is under use, and 
$X$ is an $n$-sequence of $m$ successes, then we have $p\left(X \mid \theta, \theta_{a}\right)=\theta^{\theta_{a} m}(1-\theta)^{\theta_{a}(n-m)}$. Assume that the prior pdf follows $B(\alpha, \beta)$, then the posterior pdf becomes $B\left(\alpha+\theta_{a} m, \beta+\theta_{a}(n-m)\right)$. It is clear that the value $\theta_{a}$ determines to what extent the data $X$ influences the posterior. If $\theta_{a}=0$, which means that the advisor is totally distrusted, then the posterior is equivalent with the prior, indicating that the data provided by this advisor brings no new information on the trustworthiness of the trustee.

\subsubsection{A potential capability to deal with data sparsity}

The issue of data sparsity often appears in a very large network. For example, for some large e-commerce sites, the number of sellers and buyers is so large that a buyer can hardly meet the same seller [133]. In [134], Resnick and Zeckhauser conduct an empirical study using eBay transaction data within a time period of five-month. They show that $89.0 \%$ out of all seller-buyer pairs conducted only one transaction and $98.9 \%$ conducted less than four. It implies that the seller's trustworthiness is mostly evaluated based on a single transaction record

by the buyers. In [135], Zhou and Hwang also confirm that it is extremely rare to find a node with a large number of feedbacks in a dynamically growing P2P network, because most of the network nodes only receive a few feedbacks.

Most approaches work under a basic assumption that two agents can repeatedly interact with each other, which may be the case for a small-size network. Because of the aforementioned data sparsity issue, these approaches are likely to fail to provide a satisfactory performance for large-scale networks.

As discussed in previous sections, the GBT perspective provides a theoretically sound way to analyze and integrate various typed observations, such as binary data, multi-valued data, subjective opinions, based on a consistent modeling language. To conquer problems resulted from data sparsity, we may leverage the power of GBT to take into account of social data, e.g., roles of and relationships between participating peers, and subject opinions of domain experts, in forming a more accurate trust assessment. 


\subsection{On limitations of GBT in trust modeling}

As a data-driven modeling framework, the success of a GBT based solution may depend crucially on the quality and richness of data and domain knowledge available. For example, if some third-party advisors provide unfair ratings of the trustee, then it requires that there be enough peers who offer honest ratings to override the effect of unfair ones. Provided that there is no other social metadata or domain knowledge available for use, a GBT based solution is likely to fail to produce an accurate trust evaluation due to shortage of honest rating data.

As discussed in Section 7.1.4, GBT has the potential capability to handle data sparsity, while its working requires preexisting social relationships among the network peers or domain knowledge about the network. But they may not exist in practical cases. For such cases, concrete security mechanisms, such as methods based on credentials and policies, may be the only way to establish a trust relationship between peers.

Another limitation of GBT lies in a heavy computation and memory burden caused by running PF in scenarios that demand lightweight algorithms. As a sequential sampling based approximation method, the computation and memory burden of PF is linearly dependant on the sample size. Thus, to reduce computation burden, the sample size needs to be controlled elaborately, otherwise, the accuracy of PF estimation will deteriorate severely. So unless the PF theory itself makes progress, the application scope of GBT based solutions are restricted. Another choice to get rid of heavy computation burden of PF is to employ a conjugate pair of prior pdf and likelihood function, while this may lead to performance deterioration due to model mismatch. To conquer the above limitation, the fundamental solution should come from advances in semiconductor technology that can enable a network agent to perform more computations and possess larger capacities in the memory. 


\subsection{Open research questions}

\subsubsection{How to set an appropriate initial prior pdf of trust to start the computa-}

tion engine

Running of any algorithm in a Bayesian flavor requires a pre-setting of the initial pdf, namely, $p\left(\theta_{0}\right)$ in the GBT framework. On one side, we hope that this prior pdf can reflect accurately the trustor's prior knowledge on the trustworthiness of the trustee, especially for cases in which the trustor has inadequate observations in generating the posterior estimate from the prior. On the other side, we hope that a prior pdf conjugate with the likelihood function can be used since this can make the posterior inference analytically tractable. Thus, how to balance the requirement of representation accuracy and that of computational tractability in setting the prior pdfs remains the first open question to be resolved.

\subsubsection{How to characterize multi-faceted and differentiated sides of trust in a model}

As a complicated concept, trust itself usually has multi-faceted and differentiated sides in practice. For instance, an overall trust of a WSN system can be decomposed into two components, termed communication trust and data trust, representing two different aspects of the system trust [50]. The question is how to evaluate the trustworthiness of a system by taking account of the trustworthiness of the subsystems or atomic components and the uncertainty associated with this information [136]. The consideration of the trustworthiness of the subsystems may be independent from how these trust values are assessed. The challenge lies in that, by piecing together trustworthiness of the separate entities that compose a system, we usually can not get a result that describes the trustworthiness of the whole system. Indeed, one of the major factors that influence the trust in a system is the architecture of the system, which can not be reflected by trustworthiness of the separate entities. In spite of a few application-specific attempts in the literature, e.g., methods based on the Bayesian network [133, 82, 137], there lacks a generic strategy or theory to 
address the above question, especially for complex network systems.

\subsubsection{How to connect trust with decision and action}

The trust models provide a formal language for discussing interactions between agents, while, given a trust model, a natural question remains unaddressed, namely, in general terms, how to choose decisions or actions for the trustor based on the result of trust inference. In a principled way, we can resort

to decision theory [138], which states that a rational agent should always act to maximize its expected utility (EU). We use $U: \Theta \rightarrow \mathbb{R}$ to denote a utility function, for which higher values indicate more preferred outcomes, and vice versa, then EU is calculated as follows

$$
\mathrm{EU}=\int U(\Theta) p(\Theta) d \Theta
$$

An appropriate utility function is required to be prescribed beforehand according to the trustor's domain-specific goals and preferences.

\subsubsection{How to characterize trust transfer across different domains in a model}

The phenomenon of trust transfer across domains is common in real life. For example, a middle school student who is proficient in math is likely to be also proficient in physics. It indicates that our trust in this student in terms of physics can be transferred from that in terms of math. While the notion of trust transfer is intuitively easy to comprehend, it has not been modeled formally in the literature. We argue that trust transfer is a concept that is closely related with the notion of multi-faceted and differentiated trust since trust in terms of a specific domain can be interpreted as one aspect of the system trust, which is defined across all domains considered.

\section{Conclusion}

This paper surveys trust models from a Bayesian perspective by systematizing the knowledge from a long list of trust-related research articles. Specifically, 
a general perspective for trust modeling and evaluation termed GBT is highlighted. It is shown that all models under survey can cast into a GBT based framework. The connections from each surveyed model to the GBT framework are clarified. Both capabilities and limitations of the GBT perspective are discussed, and a number of open research questions are pointed out. Despite the complex character of trust, here we make an attempt to promote the accumulation rather than the fragmentation of theory and research on trust, hoping that this may be helpful for stimulating further and deeper research on trust modeling for networked agents.

\section{Acknowledgement}

This work was partly supported by the National Natural Science Foundation of China (Nos.61571238 and 61572263).

\section{References}

[1] D. H. McKnight and N. L. Chervany, "The meanings of trust," Technical Report MISRC Working Paper Series, vol. 96, no. 4, 1996.

[2] S. Ba and P. Pavlou, "Evidence of the effect of trust building technology in electronic markets: Price premiums and buyer behavior," MIS quarterly, pp. 243-268, 2002.

[3] D. Gambetta, Trust: Making and breaking cooperative relations, Blackwell Pub, Oxford, England, 1990.

[4] D. H. McKnight, L. L. Cummings, and N. L. Chervany, Trust formation in new organizational relationships, University of Minnesota, 1996.

[5] D. Gambetta, "Can we trust trust?," in Trust: Making and breaking cooperative relations. Blackwell, 1998, pp. 213-237. 
[6] A. Kini and J. Choobineh, "Trust in electronic commerce: definition and theoretical considerations," in Proc. of the 31st Hawaii International Conference on System Sciences. IEEE, 1998, vol. 4, pp. 51-61.

[7] D. M. Rousseau, S. B. Sitkin, R. S. Burt, and C. F. Camerer, "Not so different after all: A cross-discipline view of trust," Academy of Management Review, vol. 23, no. 3, pp. 393-404, 1998.

[8] J. Cho, K. Chan, and S. Adali, "A survey on trust modeling," $A C M$ Computing Surveys, vol. 48, no. 2, pp. 28, 2015.

[9] V. Cahill, E. Gray, J.-M. Seigneur, C.D. Jensen, Y. Chen, B. Shand, N. Dimmock, A. Twigg, J. Bacon, C. English, W. Wagealla, S. Terzis, P. Nixon, G. Di Marzo Serugendo, C. Bryce, M. Carbone, K. Krukow, and M. Nielson, "Using trust for secure collaboration in uncertain environments," IEEE pervasive computing, vol. 2, no. 3, pp. 52-61, 2003.

[10] A. Selvaraj and S. Sundararajan, "Evidence-based trust evaluation system for cloud services using fuzzy logic," International Journal of Fuzzy Systems, vol. 19, no. 2, pp. 329-337, 2017.

[11] N. Rafique, M. A. Khan, N. A. Saqib, F. Bashir, C. Beard, and Z. Li, "Black hole prevention in vanets using trust management and fuzzy logic analyzer," International Journal of Computer Science and Information Security, vol. 14, no. 9, pp. 1226, 2016.

[12] M. Nagy, M. Vargas-Vera, and E. Motta, "Multi agent trust for belief combination on the semantic web," in The 4 th International Conference on Intelligent Computer Communication and Processing (ICCP). IEEE, 2008, pp. 261-264.

[13] M. Lesani and S. Bagheri, "Fuzzy trust inference in trust graphs and its application in semantic web social networks," in World Automation Congress (WAC). IEEE, 2006, pp. 1-6. 
[14] H. Chen, S. Yu, J. Shang, C. Wang, and Z. Ye, "Comparison with several fuzzy trust methods for p2p-based system," in International Conference on Information Technology and Computer Science (ITCS). IEEE, 2009, vol. 2, pp. 188-191.

[15] H. Liao, Q. Wang, and G. Li, "A fuzzy logic-based trust model in grid," in International Conference on Networks Security, Wireless Communications and Trusted Computing (NSWCTC). IEEE, 2009, vol. 1, pp. 608614.

[16] J. Luo, X. Liu, Y. Zhang, D. Ye, and Z. Xu, "Fuzzy trust recommendation based on collaborative filtering for mobile ad-hoc networks," in The 33rd IEEE Conference on Local Computer Networks (LCN). Citeseer, 2008, pp. 305-311.

[17] D. W. Manchala, "Trust metrics, models and protocols for electronic commerce transactions," in Proceedings of the 18th International Conference on Distributed Computing Systems. IEEE, 1998, pp. 312-321.

[18] S. Nefti, F. Meziane, and K. Kasiran, "A fuzzy trust model for ecommerce," in Proceedings of the 7th IEEE International Conference on E-Commerce Technology (CEC). IEEE, 2005, pp. 401-404.

[19] A. Jøsang, "Bayesian reputation systems," in Subjective Logic, pp. 289302. Springer, 2016.

[20] J. Jiang, G. Han, F. Wang, L. Shu, and M. Guizani, "An efficient distributed trust model for wireless sensor networks," IEEE transactions on parallel and distributed systems, vol. 26, no. 5, pp. 1228-1237, 2015.

[21] F. Z. Filali and B. Yagoubi, "Global trust: A trust model for cloud service selection," International Journal of Computer Network and Information Security, vol. 7, no. 5, pp. 41, 2015.

[22] N. Alhadad, Y. Busnel, P. Serrano-Alvarado, and P. Lamarre, "Trust evaluation of a system for an activity with subjective logic," in International 
Conference on Trust, Privacy and Security in Digital Business. Springer, 2014, pp. 48-59.

[23] M. Ahmadi, M. Gharib, F. Ghassemi, and A. Movaghar, "Probabilistic key pre-distribution for heterogeneous mobile ad hoc networks using subjective logic," in The 29th IEEE International Conference on Advanced Information Networking and Applications (AINA). IEEE, 2015, pp. 185192.

[24] F. Cerutti, L. M. Kaplan, T. J. Norman, N. Oren, and A. Toniolo, "Subjective logic operators in trust assessment: an empirical study," Information Systems Frontiers, vol. 17, no. 4, pp. 743-762, 2015.

[25] G. Liu, Q. Yang, H. Wang, X. Lin, and M.P. Wittie, "Assessment of multi-hop interpersonal trust in social networks by three-valued subjective logic," in Proceedings of IEEE International Conference on Computer Communications (INFOCOM). IEEE, 2014, pp. 1698-1706.

[26] A. Jøsang, "An algebra for assessing trust in certification chains," in The Network and Distributed System Security Symposium (NDSS), 1999, vol. 99, pp. 80-89.

[27] A. Jøsang, "A logic for uncertain probabilities," International Journal of Uncertainty, Fuzziness and Knowledge-Based Systems, vol. 9, no. 03, pp. 279-311, 2001.

[28] C. Lioma, B. Larsen, H. Schütze, and P. Ingwersen, "A subjective logic formalisation of the principle of polyrepresentation for information needs," in Proceedings of the 3rd symposium on Information interaction in context. ACM, 2010, pp. 125-134.

[29] N. Oren, T. J. Norman, and A. Preece, "Subjective logic and arguing with evidence," Artificial Intelligence, vol. 171, no. 10-15, pp. 838-854, 2007.

[30] R Deepa and S Swamynathan, "A trust model for directory-based service discovery in mobile ad hoc networks," in International Conference on 
Security in Computer Networks and Distributed Systems. Springer, 2014, pp. $115-126$.

[31] J. Wang and H. Sun, "Inverse problem in dsmt and its applications in trust management," in The 1st International Symposium on Data, Privacy, and E-Commerce (ISDPE). IEEE, 2007, pp. 424-428.

[32] K. Wang and M. Wu, "A trust approach for node cooperation in manet," in International Conference on Mobile Ad-Hoc and Sensor Networks. Springer, 2007, pp. 481-491.

[33] W. Zhang, S. Zhu, J. Tang, and N. Xiong, "A novel trust management scheme based on dempster-shafer evidence theory for malicious nodes detection in wireless sensor networks," The Journal of Supercomputing, pp. $1-23,2017$.

[34] V. Nguyen and V. Huynh, "Integrating with social network to enhance recommender system based-on dempster-shafer theory," in International Conference on Computational Social Networks. Springer, 2016, pp. 170181.

[35] C. Esposito, A. Castiglione, and F. Palmieri, "Information theoretic-based detection and removal of slander and/or false-praise attacks for robust trust management with dempster-shafer combination of linguistic fuzzy terms," Concurrency and Computation: Practice and Experience, 2018.

[36] P. Resnick, K. Kuwabara, R. Zeckhauser, and E. Friedman, "Reputation systems," Communications of the ACM, vol. 43, no. 12, pp. 45-48, 2000.

[37] A. Abdul-Rahman and S. Hailes, "Supporting trust in virtual communities," in Proceedings of the 33rd Hawaii International Conference on System Sciences. IEEE, 2000, vol. 6, pp. 6007-6016.

[38] C. M. Jonker and J. Treur, "Formal analysis of models for the dynamics of trust based on experiences," in European Workshop on Modelling Autonomous Agents in a Multi-Agent World. Springer, 1999, pp. 221-231. 
[39] C.M. Jonker, J. Schalken, J. Theeuwes, and J. Treur, "Human experiments in trust dynamics," in International Conference on Trust Management. Springer, 2004, pp. 206-220.

[40] S. Buchegger and J.-Y. Le Boudec, "A robust reputation system for peerto-peer and mobile ad-hoc networks," in The 2nd Workshop on Economics of Peer-to-Peer Systems (P2PEcon), 2004, pp. 1-6.

[41] Asad Amir Pirzada and Chris McDonald, "Establishing trust in pure ad-hoc networks," in Proceedings of the 27th Australasian conference on Computer Science. Australian Computer Society, Inc., 2004, vol. 26, pp. $47-54$.

[42] J. Sabater and C. Sierra, "REGRET: reputation in gregarious societies," in Proceedings of the 5th international conference on Autonomous agents. ACM, 2001, pp. 194-195.

[43] Y. Wang and V. Varadharajan, "Two-phase peer evaluation in p2p ecommerce environments," in Proceedings of IEEE International Conference on e-Technology, e-Commerce and e-Service (EEE). IEEE, 2005, pp. 654-657.

[44] F. Azzedin and M. Maheswaran, "Evolving and managing trust in grid computing systems," in IEEE Canadian Conference on Electrical and Computer Engineering (CCECE). IEEE, 2002, vol. 3, pp. 1424-1429.

[45] K. Hung, K. Lui, and Y. Kwok, "A trust-based geographical routing scheme in sensor networks," in IEEE Wireless communications and networking conference (WCNC). IEEE, 2007, pp. 3123-3127.

[46] W. Song and V. Phoha, "Neural network-based reputation model in a distributed system," in Proceedings of IEEE International Conference on e-Commerce Technology (CEC). IEEE, 2004, pp. 321-324.

[47] H. Baohua, H. Heping, and L. Zhengding, "Identifying local trust value with neural network in $\mathrm{P} 2 \mathrm{P}$ environment," in The First IEEE and IFIP 
International Conference in Central Asia on Internet. IEEE, 2005, pp. $1-5$.

[48] S. Songsiri, "MTrust: a reputation-based trust model for a mobile agent system," in International Conference on Autonomic and Trusted Computing. Springer, 2006, pp. 374-385.

[49] Y. Wang, V. Cahill, E. Gray, C. Harris, and L. Liao, "Bayesian network based trust management," in International Conference on Autonomic and Trusted Computing. Springer, 2006, pp. 246-257.

[50] M. Momani, S. Challa, and R. Alhmouz, "BNWSN: Bayesian network trust model for wireless sensor networks," in Mosharaka International Conference on Communications, Computers and Applications (MIC-CCA). IEEE, 2008, pp. 110-115.

[51] C. T. Nguyen, O. Camp, and S. Loiseau, "A bayesian network based trust model for improving collaboration in mobile ad hoc networks," in IEEE International Conference on Research, Innovation and Vision for the Future. IEEE, 2007, pp. 144-151.

[52] P. Michiardi and R. Molva, "Core: a collaborative reputation mechanism to enforce node cooperation in mobile ad hoc networks," in Advanced communications and multimedia security, pp. 107-121. Springer, 2002.

[53] L. Xiong and L. Liu, "A reputation-based trust model for peer-topeer e-commerce communities," in IEEE International Conference on E-Commerce (CEC). IEEE, 2003, pp. 275-284.

[54] T. Jiang and J.S. Baras, "Ant-based adaptive trust evidence distribution in MANET," in Proceedings of the 24th International Conference on Distributed Computing Systems Workshops. IEEE, 2004, pp. 588-593.

[55] W. Wang, G. Zeng, and L. Yuan, "Ant-based reputation evidence distribution in p2p networks," in The 5th International Conference Grid and Cooperative Computing (GCC). IEEE, 2006, pp. 129-132. 
[56] F.G. Mármol and G.M. Pérez, "Providing trust in wireless sensor networks using a bio-inspired technique," Telecommunication systems, vol. 46, no. 2, pp. 163-180, 2011.

[57] F.G. Marmol, G.M. Perez, and A. Skarmeta, "TACS, a trust model for P2P networks," Wireless Personal Communications, vol. 51, no. 1, pp. 153-164, 2009.

[58] N. Santos, R. Rodrigues, K.P. Gummadi, and S. Saroiu, "Policy-sealed data: A new abstraction for building trusted cloud services.," in USENIX security symposium, 2012, pp. 175-188.

[59] B.C. Neuman and T. Ts'o, "Kerberos: An authentication service for computer networks," IEEE Communications magazine, vol. 32, no. 9, pp. 33-38, 1994.

[60] M. Winslett, T. Yu, K.E. Seamons, A. Hess, J. Jacobson, R. Jarvis, B. Smith, and L. Yu, "Negotiating trust in the web," IEEE Internet Computing, vol. 6, no. 6, pp. 30-37, 2002.

[61] N. Li, W.H. Winsborough, and J.C. Mitchell, "Distributed credential chain discovery in trust management," Journal of Computer Security, vol. 11, no. 1, pp. 35-86, 2003.

[62] W. Nejdl, D. Olmedilla, and M. Winslett, "Peertrust: Automated trust negotiation for peers on the semantic web," in Workshop on Secure Data Management. Springer, 2004, pp. 118-132.

[63] P. Bonatti and D. Olmedilla, "Driving and monitoring provisional trust negotiation with metapolicies," in The 6th IEEE International Workshop on Policies for Distributed Systems and Networks. IEEE, 2005, pp. 14-23.

[64] W.H. Winsborough, K.E. Seamons, and V.E. Jones, "Automated trust negotiation," in Proceedings of DARPA Information Survivability Conference and Exposition (DISCEX). IEEE, 2000, vol. 1, pp. 88-102. 
[65] M.Y. Becker and P. Sewell, "Cassandra: Distributed access control policies with tunable expressiveness," in Proceedings of the 5th IEEE International Workshop on Policies for Distributed Systems and Networks (POLICY). IEEE, 2004, pp. 159-168.

[66] D. Olmedilla, "Security and privacy on the semantic web," in Security, Privacy, and Trust in Modern Data Management, pp. 399-415. Springer, 2007.

[67] M.K. Lee and E. Turban, "A trust model for consumer internet shopping," International Journal of electronic commerce, vol. 6, no. 1, pp. 75-91, 2001.

[68] G. Theodorakopoulos and J.S. Baras, "On trust models and trust evaluation metrics for ad hoc networks," IEEE Journal on selected areas in Communications, vol. 24, no. 2, pp. 318-328, 2006.

[69] F. Bao, R. Chen, M. Chang, and J.-H. Cho, "Hierarchical trust management for wireless sensor networks and its applications to trust-based routing and intrusion detection," IEEE Transactions on network and service management, vol. 9, no. 2, pp. 169-183, 2012.

[70] A. Boukerche and Y. Ren, "A trust-based security system for ubiquitous and pervasive computing environments," Computer Communications, vol. 31, no. 18, pp. 4343-4351, 2008.

[71] S.D. Kamvar, M.T. Schlosser, and H. Garcia-Molina, "The eigentrust algorithm for reputation management in p2p networks," in Proceedings of the 12th international conference on World Wide Web. ACM, 2003, pp. 640-651.

[72] L. Xiong and L. Liu, "Peertrust: Supporting reputation-based trust for peer-to-peer electronic communities," IEEE Transactions on Knowledge and Data Engineering, vol. 16, no. 7, pp. 843-857, 2004. 
[73] K. Regan and R. Cohen, "A model of indirect reputation assessment for adaptive buying agents in electronic markets," Proceedings of the Business Agents and Semantic Web (BASeWEB), pp. 41-51, 2005.

[74] G. Zacharia and P. Maes, "Trust management through reputation mechanisms," Applied Artificial Intelligence, vol. 14, no. 9, pp. 881-907, 2000.

[75] X. Su, M. Zhang, Y. Mu, and K.M. Sim, "PBTrust: A priority-based trust model for service selection in general service-oriented environments," in IEEE/IFIP 8th International Conference on Embedded and Ubiquitous Computing (EUC). IEEE, 2010, pp. 841-848.

[76] C. Zouridaki, "Trust establishment for reliable data packet delivery in mobile ad hoc networks," 2007.

[77] B. Liu, Z. Xu, J. Chen, and G. Yang, "Toward reliable data analysis for internet of things by bayesian dynamic modeling and computation," in IEEE China Summit and International Conference on Signal and Information Processing (ChinaSIP). IEEE, 2015, pp. 1027-1031.

[78] B. Liu and G. Yang, "Probabilistic trust evaluation with inaccurate reputation reports," International Journal of Distributed Sensor Networks, vol. 11 , no. 6 , pp. 1-7, 2015.

[79] M.K. Denko, T. Sun, and I. Woungang, "Trust management in ubiquitous computing: A Bayesian approach," Computer Communications, vol. 34, pp. 398-406, 2011.

[80] Bin Liu and Shi Cheng, "State space model based trust evaluation over wireless sensor networks: An iterative particle filter approach," Journal of Engineering, vol. 2017, no. 4, pp. 101-109, 2017.

[81] R. Chen, F. Bao, M. Chang, and J.-H. Cho, "Dynamic trust management for delay tolerant networks and its application to secure routing," IEEE Transactions on Parallel and Distributed Systems, vol. 25, no. 5, pp. 12001210, 2014. 
[82] Y. Wang and J. Vassileva, "Bayesian network-based trust model," in Proceedings of IEEE/WIC International Conference on Web Intelligence (WI). IEEE, 2003, pp. 372-378.

[83] J. Dubey and V. Tokekar, "Bayesian network based trust model with time window for pure P2P computing systems," in IEEE Global Conference on Wireless Computing and Networking (GCWCN). IEEE, 2014, pp. 219223.

[84] M. Venanzi, W.L. Teacy, A. Rogers, and N.R. Jennings, "Bayesian modelling of community-based multidimensional trust in participatory sensing under data sparsity.," in IJCAI, 2015, pp. 717-724.

[85] A. Xu and G. Dudek, "Optimo: Online probabilistic trust inference model for asymmetric human-robot collaborations," in Proceedings of the 10th Annual ACM/IEEE International Conference on Human-Robot Interaction. ACM, 2015, pp. 221-228.

[86] J. Guo, R. Chen, and J. Tsai, "A survey of trust computation models for service management in internet of things systems," Computer Communications, vol. 97, pp. 1-14, 2017.

[87] Z. Yan, P. Zhang, and A.V. Vasilakos, "A survey on trust management for Internet of things," Journal of network and computer applications, vol. 42, pp. 120-134, 2014.

[88] A. Jøsang, R. Ismail, and C. Boyd, "A survey of trust and reputation systems for online service provision," Decision support systems, vol. 43, no. 2, pp. 618-644, 2007.

[89] J. Zhang, "A survey on trust management for vanets," in IEEE international conference on Advanced information networking and applications (AINA). IEEE, 2011, pp. 105-112. 
[90] L. Viljanen, "Towards an ontology of trust," in International Conference on Trust, Privacy and Security in Digital Business. Springer, 2005, pp. $175-184$.

[91] D. Artz and Y. Gil, "A survey of trust in computer science and the semantic web," Web Semantics: Science, Services and Agents on the World Wide Web, vol. 5, no. 2, pp. 58-71, 2007.

[92] H. Yu, Z. Shen, C. Miao, C. Leung, and D. Niyato, "A survey of trust and reputation management systems in wireless communications," Proceedings of the IEEE, vol. 98, no. 10, pp. 1755-1772, 2010.

[93] M. Momani and S. Challa, "Survey of trust models in different network domains," International Journal of Ad Hoc and Ubiquitous Computing, vol. 1, no. 3, pp. 1-19, 2010.

[94] M. Momani, "Trust models in wireless sensor networks: A survey," in International Conference on Network Security and Applications. Springer, 2010, pp. 37-46.

[95] T. Grandison and M. Sloman, "A survey of trust in internet applications," IEEE Communications Surveys $\&$ Tutorials, vol. 3, no. 4, pp. 2-16, 2000.

[96] W. Sherchan, S. Nepal, and C. Paris, "A survey of trust in social networks," ACM Computing Surveys, vol. 45, no. 4, pp. 47, 2013.

[97] I. Pinyol and J. Sabater-Mir, "Computational trust and reputation models for open multi-agent systems: a review," Artificial Intelligence Review, vol. 40, no. 1, pp. 1-25, 2013.

[98] J. Lopez, R. Roman, I. Agudo, and C. Fernandez-Gago, "Trust management systems for wireless sensor networks: Best practices," Computer Communications, vol. 33, no. 9, pp. 1086-1093, 2010.

[99] M. S. Arulampalam, S. Maskell, N. Gordon, and T. Clapp, "A tutorial on particle filters for online nonlinear/non-Gaussian Bayesian tracking," IEEE Trans. on Signal Processing, vol. 50, no. 2, pp. 174-188, 2002. 
[100] B. Liu, "Instantaneous frequency tracking under model uncertainty via dynamic model averaging and particle filtering," IEEE Trans. on Wireless Communications, vol. 10, no. 6, pp. 1810-1819, 2011.

[101] B. Liu, C. Ji, Y. Zhang, C. Hao, and K. Wong, "Multi-target tracking in clutter with sequential Monte Carlo methods," IET radar, sonar \& navigation, vol. 4, no. 5, pp. 662-672, 2010.

[102] B. Liu and C. Hao, "Sequential bearings-only-tracking initiation with particle filtering method," The Scientific World Journal, vol. 2013, pp. $1-7,2013$.

[103] B. Liu, X. Ma, and C. Hou, "A particle filter using SVD based sampling Kalman filter to obtain the proposal distribution," in Proc. of IEEE Conf. on Cybernetics and Intelligent Systems. IEEE, 2008, pp. 581-584.

[104] B. Liu, "Robust particle filter by dynamic averaging of multiple noise models," in Proc. of the 42nd IEEE Int'l Conf. on Acoustics, Speech, and Signal Processing (ICASSP). IEEE, 2017, pp. 4034-4038.

[105] Y. Dai and B. Liu, "Robust video object tracking via Bayesian model averaging-based feature fusion," Optical Engineering, vol. 55, no. 8, pp. $083102(1)-083102(11), 2016$.

[106] A. Jøsang and R. Ismail, "The beta reputation system," in Proceedings of the 15th BLED Electronic Commerce Conference, 2002, vol. 5, pp. 25022511.

[107] W.L. Teacy, J. Patel, N.R. Jennings, and M. Luck, "Coping with inaccurate reputation sources: Experimental analysis of a probabilistic trust model," in Proceedings of the 4th International Joint Conference on Autonomous Agents and Multiagent Systems. ACM, 2005, pp. 997-1004.

[108] W.L. Teacy, J. Patel, N.R. Jennings, and M. Luck, "Travos: Trust and reputation in the context of inaccurate information sources," Autonomous Agents and Multi-Agent Systems, vol. 12, no. 2, pp. 183-198, 2006. 
[109] T.D. Huynh, N.R. Jennings, and N.R. Shadbolt, "An integrated trust and reputation model for open multi-agent systems," Autonomous Agents and Multi-Agent Systems, vol. 13, no. 2, pp. 119-154, 2006.

[110] J. Patel, W.L. Teacy, N.R. Jennings, and M. Luck, "A probabilistic trust model for handling inaccurate reputation sources," in International Conference on Trust Management. Springer, 2005, pp. 193-209.

[111] W.L. Teacy, N.R. Jennings, A. Rogers, and M. Luck, "A hierarchical bayesian trust model based on reputation and group behaviour," in Proc. of 6th European Workshop on Multi-Agent Systems, 2008, pp. 1-15.

[112] A. Whitby, A. Jøsang, and J. Indulska, "Filtering out unfair ratings in bayesian reputation systems," in Proceedings of the 7th International Workshop on Trust in Agent Societies, 2004, vol. 6, pp. 106-117.

[113] J. Zhang and R. Cohen, "Evaluating the trustworthiness of advice about seller agents in e-marketplaces: A personalized approach," Electronic Commerce Research and Applications, vol. 7, no. 3, pp. 330-340, 2008.

[114] L. Mui, M. Mohtashemi, and A. Halberstadt, "A computational model of trust and reputation," in Proceedings of the 35th Annual Hawaii International Conference on System Sciences (HICSS). IEEE, 2002, pp. 24312439 .

[115] Z. Despotovic and K. Aberer, "P2p reputation management: Probabilistic estimation vs. social networks," Computer Networks, vol. 50, no. 4, pp. 485-500, 2006.

[116] M. Nielsen, K. Krukow, and V. Sassone, "A bayesian model for eventbased trust," Electronic Notes in Theoretical Computer Science, vol. 172, pp. 499-521, 2007.

[117] S. Reece, A. Rogers, S. Roberts, and N.R. Jennings, "Rumours and reputation: Evaluating multi-dimensional trust within a decentralised repu- 
tation system," in Proceedings of the 6th International Joint Conference on Autonomous Agents and Multiagent Systems. ACM, 2007, p. 165.

[118] K. Regan, P. Poupart, and R. Cohen, "Bayesian reputation modeling in e-marketplaces sensitive to subjectivity, deception and change," in Proceedings of the National Conference on Artificial Intelligence. Menlo Park, CA; Cambridge, MA; London; AAAI Press; MIT Press; 1999, 2006, vol. 21, p. 1206.

[119] J. Wang and B. Liu, "Online fault-tolerant dynamic event region detection in sensor networks via trust model," in IEEE Wireless Communications and Networking Conference (WCNC). IEEE, 2017, pp. 1-6.

[120] A. Jøsang, "Artificial reasoning with subjective logic," in Proceedings of the 2nd Australian Workshop on Commonsense Reasoning. Citeseer, 1997, vol. 48, p. 34 .

[121] M. Ivanovska, A. Jøsang, J. Zhang, and S. Chen, "Joint subjective opinions," in Modeling Decisions for Artificial Intelligence. Springer, 2017, pp. 220-233.

[122] S. Pope and A. Jøsang, "Analysis of competing hypotheses using subjective logic," Tech. Rep., QUEENSLAND UNIV BRISBANE (AUSTRALIA), 2005.

[123] M. Ivanovska, A. Jøsang, and F. Sambo, "Bayesian deduction with subjective opinions.," in The 15th International Conference on Principles of Knowledge Representation and Reasoning (KR), 2016, pp. 484-493.

[124] A. Jøsang, "Conditional reasoning with subjective logic," Journal of Multiple-Valued Logic and Soft Computing, vol. 15, no. 1, pp. 5-38, 2008.

[125] A. Jøsang and R. Hankin, "Interpretation and fusion of hyper opinions in subjective logic," in The 15th International Conference on Information Fusion (FUSION). IEEE, 2012, pp. 1225-1232. 
[126] A. Jøsang, D. Wang, and J. Zhang, "Multi-source fusion in subjective logic," in Proceedings of FUSION, 2017.

[127] A. Jøsang and L. Kaplan, "Principles of subjective networks," in The 19th International Conference on Information Fusion (FUSION). IEEE, 2016, pp. 1292-1299.

[128] A. Jøsang, R. Hayward, and S. Pope, "Trust network analysis with subjective logic," in Proceedings of the 29th Australasian Computer Science Conference. Australian Computer Society, Inc., 2006, vol. 48, pp. 85-94.

[129] A. Jøsang and T. Bhuiyan, "Optimal trust network analysis with subjective logic," in The 2nd International Conference on Emerging Security Information, Systems and Technologies (SECURWARE). IEEE, 2008, pp. 179-184.

[130] Y. Liu, K. Li, Y. Jin, Y. Zhang, and W. Qu, "A novel reputation computation model based on subjective logic for mobile ad hoc networks," Future Generation Computer Systems, vol. 27, no. 5, pp. 547-554, 2011.

[131] L. Kaplan and M. Ivanovska, "Efficient subjective bayesian network belief propagation for trees," in The 19th International Conference on Information Fusion (FUSION). IEEE, 2016, pp. 1-8.

[132] L. Kaplan, M. Sensoy, Y. Tang, S. Chakraborty, C. Bisdikian, and G. de Mel, "Reasoning under uncertainty: Variations of subjective logic deduction," in 16th International Conference on Information Fusion (FUSION). IEEE, 2013, pp. 1910-1917.

[133] Y. Wang and J. Vassileva, "Bayesian network trust model in peer-to-peer networks," in Moro G., Sartori C., Singh M.P. (eds) Agents and Peerto-Peer Computing (AP2PC 2003). Lecture Notes in Computer Science. 2003, vol. 2872, pp. 23-34, Springer-Verlag. 
[134] P. Resnick and R. Zeckhauser, "Trust among strangers in internet transactions: Empirical analysis of ebay's reputation system," Adv. Appl. Microeconomics, vol. 11, pp. 127-157, 2002.

[135] R. Zhou and K. Hwang, "Powertrust: A robust and scalable reputation system for trusted peer-to-peer computing," IEEE Transactions on Parallel and Distributed Systems, vol. 18, no. 4, pp. 460-473, 2007.

[136] S. Ries, S. M. Habib, Mühlhäuser M., and V. Varadharajan, "Certainlogic: A logic for modeling trust and uncertainty," in International Conference on Trust and Trustworthy Computing. 2011, vol. 6740, pp. 254-261, Springer.

[137] Y. Wang and J. Vassileva, "Trust and reputation model in peer-to-peer networks," in Proceedings of the 3rd International Conference on Peerto-Peer Computing, 2003, pp. 150-157.

[138] J.O. Berger, Statistical decision theory and Bayesian analysis, Springer Science \& Business Media, 2013. 